\title{
EVOLUCIÓN HISTÓRICA-EPISTEMOLÓGICA DE LA ECONOMÍA CIRCULAR: ¿'HACIA UN NUEVO PARADIGMA DEL DESARROLLO?
}

\author{
HISTORIC-EPISTEMOLOGICAL EVOLUTION OF THE CIRCULAR \\ ECONOMY: TOWARDS A NEW DEVELOPMENT PARADIGM?
}

\section{EVOLUÇÃO HISTÓRICO-EPISTEMOLÓGICA DA ECONOMIA CIRCULAR: EM DIREÇÃO A UM NOVO PARADIGMA DE DESENVOLVIMENTO?}

\section{Oscar Ugalde Hernández ${ }^{1}$}

\begin{abstract}
Resumen
El desarrollo histórico-epistemológico del concepto de economía circular ha sido un proceso que inició desde la escuela clásica de Economía hacia finales del siglo XVIII, y que ha llegado a consolidarse hasta las primeras dos décadas del siglo XXI. En sus inicios, los economistas clásicos mostraron noción de las posibles consecuencias de alcanzar los límites de recursos del planeta, y llegar a un estado estacionario. Inclusive se generó preocupación por el impacto negativo del sistema económico convencional de mercado en el ambiente. Tal preocupación se profundizó en el siglo $\mathrm{XX}$, donde surge un movimiento ambientalista contracultura que germina con ideas innovadoras para poder regenerar y restaurar nuestros recursos, con base en cómo los sistemas naturales funcionan. Por tanto, surge la economía circular en el siglo XXI en calidad de un concepto tan robusto que se empieza a considerar como un nuevo paradigma del desarrollo que requiere de esquemas de acción pronta para cambiar el rumbo en el uso de los recursos finitos del planeta.
\end{abstract}

Palabras clave: sistemas económicos, medio ambiente, circularidad, desperdicio.

\begin{abstract}
The historic-epistemological development of the circular economy concept is a process that started with the classical school of economics at the end of the $18^{\text {th }}$ century and was finally consolidated during the first two decades of the $21^{\text {st }}$ century. Since its beginning, classical economists
\end{abstract}

Doi: https://doi.org/10.15359/eys.26-59.5

Recibido: 03-02-2021. Reenvíos: 14-06-2021, 21-06-2021. Aceptado: 26-06-2021. Publicado: 30-06-2021.

1 Doctor en Ciencias Económicas y Empresariales, Escuela de Relaciones Internacionales de la Universidad Nacional, Costa Rica. Correo: oscar.ugalde.hernandez@una.ac.cr, ORCID: https://orcid. org/0000-0001-8566-7821

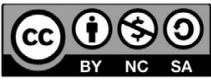


showed notion of the possible consequences of reaching the limits of the planet's resources and moving into a static state. There was even concern about the negative impact of the mainstream economic market model on the environment. Such concern increased during then 20th century, resulting in a counterculture environmental movement that brought innovative ideas to regenerate and restore our resources, based on how natural systems work. As a consequence, circular economy was born in the 21 st century as a robust concept considered to be a new development paradigm requiring prompt action schemes to change the use of the finite resources of our planet.

Keywords: economic systems, environment, circularity, waste.

\section{Resumo}

O desenvolvimento histórico-epistemológico do conceito de economia circular foi um processo que se iniciou desde a escola clássica da Economia no final do século XVIII e se consolidou até as duas primeiras décadas do século XXI. Em seus primórdios, os economistas clássicos mostraram noção das possíveis consequências de se atingir os limites dos recursos do planeta e chegar a um estado estacionário. A preocupação foi levantada até mesmo sobre o impacto negativo do sistema econômico de mercado convencional sobre o meio ambiente. Essa preocupação se aprofundou no século $X X$, quando surgiu um movimento ambientalista de contracultura, que germinou com ideias inovadoras para regenerar e restaurar nossos recursos, com base no funcionamento dos sistemas naturais. Portanto, a economia circular surge no século XXI como um conceito tão robusto que começa a ser considerada como um novo paradigma de desenvolvimento que requer esquemas de ação imediata para mudar o curso no uso dos recursos finitos do planeta.

Palavras-chave: sistemas econômicos, meio ambiente, circularidade, resíduos.

\section{Introducción}

Desde la escuela clásica de economía de finales del siglo XVIII hasta el presente, se ha obviado que el sistema económico se encuentra inmerso dentro de un entorno natural y en un medio físico. Los manuales de economía se han referido históricamente a las actividades económicas básicas como lo son: producción, distribución y consumo. Esta visión se ha ampliado con el concepto de la economía de los materiales, donde se añade la extracción y el descarte. Sin embargo, el subsistema económico se ha expandido notablemente en sus dimensiones, y el ambiente ha visto superada su capacidad asimilativa con cantidades cada vez mayores de materia y energía utilizadas. Por tanto, es indispensable replantear las conexiones que existen entre el sistema natural y la economía. 
Como catalizadores de la expansión del subsistema económico y subsecuentes alteraciones sin precedentes de los ecosistemas, se encuentran: a. la población mundial total, la cual ha crecido entre 1960 y el 2020 en un 155 por ciento, y se estima que crecerá en un 219\% para el 2050 (Grupo Banco Mundial, 2020), y, b. el cambio cualitativo y cuantitativo en la producción y el consumo durante el siglo XX y que continúa en el siglo XXI, que ha hecho que el crecimiento económico dependa de la extracción de materiales. El uso global de materiales para el año 2011 fue de "79 Gt (gigatones que es equivalente a 109 toneladas)" y se proyecta que va a llegar "a $167 \mathrm{Gt}$ en 2060", siendo un incremento de 2.1 veces. (Organization of Economic Cooperation and Development [OECD], 2018: 4). Una mayor población mundial, así como cambios significativos en sus patrones de producción y consumo, resultan también en c. un ingreso per cápita global promedio que va a aumentar "2.7 veces" entre los años 2011 y 2060 (OECD, 2018: 4).

Los modelos económicos convencionales lineales aplicados al ambiente, tal como la economía ambiental, utilizan supuestos neoclásicos tales como la asignación eficiente de recursos, la racionalidad limitada, el marginalismo, al igual que el análisis de externalidades negativas y las fallas de mercado. Sin embargo, estos no desarrollan herramientas analíticas para tener en cuenta la naturaleza agotable de la mayoría de los recursos naturales. Por esta razón, la economía circular ha surgido mucho durante los últimos quince años, porque incorpora la idea del estado estacionario manteniendo las actividades económicas dentro de las limitaciones impuestas por la naturaleza, con un sistema económico "regenerativo y restaurativo" (Ellen MacArthur Foundation, 2013:7). Para tal efecto, se plantea la siguiente pregunta de investigación:

El desarrollo histórico-epistemológico del concepto de economía circular ¿̇ha permitido lograr su consagración como un nuevo paradigma del desarrollo a la luz de nuevas ideas que destacan la necesidad de simular el comportamiento de los sistemas naturales dentro de los límites físicos del planeta?

Para abordar esta pregunta, se plantean los siguientes objetivos de investigación: 1) Realizar una revisión literaria de las escuelas de pensamiento que han contribuido a forjar el concepto de economía circular. 2) Determinar de qué manera cada escuela de pensamiento, y sus autores destacados, han contribuido al desarrollo históricoepistemológico de un nuevo paradigma de desarrollo.

\section{Aportes de la economía clásica desde finales del siglo XVIII y XIX en la comprensión de los límites del crecimiento y el estado estacionario}

En 1776 Adam Smith publica Una investigación sobre la naturaleza y las causas de la riqueza de las naciones, en la cual se analiza cómo las naciones logran alcanzar crecimiento económico gracias a la división del trabajo, a la acumulación de capital y al intercambio eficiente de bienes y servicios. Sin embargo, Smith enfatiza en que "este producto (producto anual de la tierra y el trabajo del país) puede ser muy grande, pero jamás será infinito" (Smith, 1776, s. f., p. 258). Smith (s. f.) considera a su vez que la aparición del "estado estacionario" era un acontecimiento inevitable, pero que se produciría en un futuro muy lejano. 
Hacia 1817 David Ricardo publica su libro Principios de política económica y tributación. En él se desarrolla la teoría del valor, la cual estipula que el precio de los bienes es determinado por las horas relativas de mano de obra dedicadas a su producción, asumiendo la tierra como un factor fijo, y con una intensidad del capital similar en los diversos sectores económicos. El tamaño de los beneficios empresariales es el resultado del cultivo de la tierra y de los salarios reales otorgados. Además, se desarrolla su teoría de crecimiento, la cual estipula que la prosperidad generada por más ahorros, acumulación y demanda de mano de obra lleva a un mayor crecimiento de la población, y eventualmente al uso de tierras cada vez menos fértiles para el cultivo. Al final se llegaría a un "estado estacionario" (Ricardo, 1821, 2004, capítulo 5, párr. 25), en el cual la capacidad de acumular capital e inversión desaparece como consecuencia de los rendimientos decrecientes del capital físico. A pesar de esto, Ricardo aduce que la tecnología logra aumentar el producto marginal del cultivo de la tierra, pero que a su vez introduce maquinaria que no es intensiva en mano de obra.

En un tono más pesimista que el de Adam Smith y el de David Ricado, Thomas Malthus argumenta en su Ensayo sobre el principio de la población (1798/1846) que los faltantes de medios de subsistencia llevarían inevitablemente a la especie humana a la muerte de muchos. Para Malthus, el crecimiento poblacional sucedía de forma "geométrica" (Malthus, 1798/1846, libro 1, capítulo 1, párr. 32) y los alimentos se producían de forma "aritmética" (Malthus, 1798/1846, libro 1, capítulo 1, párr. 43). Por tanto, Malthus representa el primer autor clásico en mantener una posición sombría acerca de si el planeta puede proveer los recursos suficientes para mantener un crecimiento poblacional en aumento. Se vislumbra, por primera vez, una escuela de pensamiento que se contrapone al positivismo de Smith y Ricardo, y expone los límites del crecimiento a los que podría llegar el sistema económico y, por ende, los recursos finitos del planeta.

Adicionalmente, Karl Marx (1894/1999) postula su controversial teoría del metabolismo social, en la cual argumenta que la ruptura "irreparable" en el proceso interdependiente entre la humanidad y el resto de la naturaleza se da a causa de la producción agrícola capitalista insostenible, y la creciente división entre el campo y la ciudad (Marx, 1894/1999), siendo consecuencia de la degradación ambiental generada en esta interdependencia entre las partes. Es una crítica a las prácticas agrícolas que llevan a los recursos a sus límites de producción, y es un primer acercamiento teórico a la circularidad entre los sistemas económicos y naturales.

Por su lado, John Stuart Mill desarrolla Principios de Política Económica (1848/1978), una perspectiva en donde relativiza el catastrofismo que pudiera derivarse del estado estacionario, con un acento más bien deseable de este.

Casi no sería necesario decir que una situación estacionaria del capital y de la población no implica una situación estacionaria del adelanto humano. Sería más amplio que nunca el campo para la cultura del entendimiento y para el progreso moral y social (Mill, 1848/1978: 643). 
Se forma un sentimiento de que el estado estacionario no necesariamente debe resultar perjudicial para la sociedad. Puede crear una alternativa de desarrollo novedosa como contrabalanza del sistema económico convencional. No solo la tecnología, sino otros conceptos como el "arte de vivir" (Mill, 1848/1978: 643) se convierten en ideas clave para optar a un futuro prometedor.

William Stanley Jevons publica en 1865 un estudio sobre los límites a la expansión de la economía inglesa derivados del uso de su principal recurso energético, el carbón, llamado La cuestión del carbón. Jevons (1865/2018) se enfoca en la eficiencia generada por el progreso tecnológico en términos marginales (cada nueva invención requería menos consumo de carbón para funcionar) (Polimeni et al., 2009: 48). Sin embargo, esto traería un mayor consumo de carbón en términos absolutos, al ser la nueva invención más barata de operar y utilizada para muchos otros usos. Jevons argumenta que el consumo de recursos era exponencial, y que el crecimiento poblacional inevitablemente sobrepasaría la capacidad del país para expandir sus recursos. El crecimiento económico caería, y al ser el carbón un recurso no renovable, eventualmente alcanzaría su capacidad finita.

Los filósofos económicos clásicos de los siglos XVIII y XIX demuestran que entienden la capacidad finita de los recursos del planeta; sin embargo, no se esmeran en entender sus consecuencias inmediatas como lo hacen las posturas de Smith, Ricardo y de Mill. Son Malthus, Jevons y Marx quienes evidencian los impactos a más largo plazo si los recursos del planeta son llevados al límite; sin embargo, solo Malthus y Jevons contextualizan el estado estacionario de una manera más prospectiva. Gracias al aporte realizado por estos autores, se logra comprender la necesidad que eventualmente afrontarían los sistemas económicos de buscar soluciones sostenibles y en armonía con el ambiente.

\section{Orígenes, construcción y formación de los fundamentos de la economía circular durante el siglo $\mathrm{XX}$}

A pesar de que hacia finales del siglo XIX ya se habían dado las primeras protestas contra la contaminación, y a favor de la conservación de los recursos naturales, la atención de los economistas sobre el agotamiento de los recursos naturales empezó en los años veinte con Arthur Cecil Pigou (1932/2010) en su obra The Economics of Welfare. Él argumenta que la contaminación industrial puede considerarse como una externalidad (costo impuesto no deseado) negativa para la sociedad. Se genera habitualmente cuando el precio de mercado de un bien o servicio no refleja los verdaderos costos para la sociedad, creando una falla de mercado. Pigou propuso un impuesto sobre las externalidades negativas con el objetivo de reducir su incidencia a un nivel eficiente (Pigou, 1932/2010).

Posteriormente, Harold Hotelling (1931) escribe The Economics of Exhaustible Resources. Él estipulaba que la desaparición de los inventarios de materiales, de bosques y de otros activos agotables ha llevado a crear presiones para regular su explotación. Hotelling argumenta que estos activos poseen un valor muy accesible para los consumidores, y esto repercute en el precio de los alimentos en el futuro, causando una explotación rápida y egoísta por la generación actual. Esto lleva al 
surgimiento del movimiento conservacionista como respuesta ante estas tendencias del sistema económico convencional.

Estas críticas a los efectos de la industrialización y los problemas que la misma creaba en la sociedad y en el medio ambiente continuaron más intensamente a partir de la segunda mitad del siglo XX. En la obra Primavera silenciosa (1962/2010), Rachel Carson llamaba la atención sobre el envenenamiento masivo de las aves afectadas en su ingesta por la contaminación de los agroquímicos industriales. Carson (1962/2010) insistió en adoptar una posición discordante: "... no debemos continuar siguiendo el consejo de los que nos dicen que llenemos nuestro mundo de productos químicos venenosos" (p. 292).

En otra obra, Ciencia y supervivencia (1963), Barry Commoner advertía sobre los riesgos que implicaban las políticas tecnológicas de la civilización industrial. Él denunciaba la irracionalidad a la que nos conducía lo que, a su juicio, era un infundado optimismo tecnológico. Las catástrofes y accidentes derivados de fallos en el complejo tecnocientífico, de alguna manera, confirmaron la legitimidad de su mensaje (Commoner, 1963).

Adicionalmente, Kenneth Boulding con su obra La economía de la futura nave espacial Tierra (1966/2012) enfatiza la capacidad finita del planeta para extraer recursos y asimilar residuos, al compararlo con la "nave espacial Tierra". La idea de Boulding sobre la "nave espacial Tierra" también era una forma optimista de sugerir que la humanidad misma, como el astronauta de la nave, tiene la capacidad y habilidad de dirigir responsablemente la nave, administrando sabiamente sus finitos recursos naturales para el beneficio de sus ocupantes:

Luego de pasar casi desapercibo, el movimiento ambientalista vuelve a tomar relevancia durante los años cincuenta e inicios de los sesenta como resultado de las ideas de Carson (1962), Commoner (1963) y Boulding (1966/2012). Ellos enfatizan sobre los efectos adversos y riesgos generados por la industrialización, la tecnología y el progreso económico sobre los recursos y especies de la Tierra. Estos autores aún no plantean con claridad si el estado estacionario es un resultado deseable o no, pero se insiste en que no se puede continuar por el mismo camino.

Tal esfuerzo de concientización durante la década de los sesenta también permitió el desarrollo de una contracultura (Roszak, 1969) representada en los movimientos ambientalistas utopianistas y el comunalismo de 'regreso a la naturaleza' (Roszak, 1969; Turner, 2006 citado en Crocker, Saint, Chen \& Yindong, T. (Eds), 2018).

Según Crocker (2018), esta contracultura ambientalista fue influenciada por tres grandes tendencias: a. el resurgimiento de la biología y la ecología como ciencias que podrían aportar mucho a encontrar un paradigma de desarrollo alternativo, resultante del pesimismo generado por la amenaza nuclear de la Guerra Fría, los desastres a causa de la industrialización, e inclusive el pensamiento neo-malthusiano de escasez de recursos y combustibles de Erlich en su libro La bomba de población (1968); b. las dimensiones sociales de la nuevas tecnologías de la época. Autores como Turner (2006) criticaron a la sociedad tecnocrática del momento al considerar que tenía una opinión utópica en 
relación con las primeras computadoras y comunicaciones modernas de bajo costo; c. el diseño ecológico, encabezado por los arquitectos paisajistas McHarg (1969) con su idea de diseño natural, y Lyle (1994) con su diseño regenerativo. Consiste en diseñar las cosas inspirados en la naturaleza, el lugar y la comunidad (Formia, 2017). Estos conceptos siembran la semilla que posteriormente germinaría en el enfoque "de la cuna a la cuna" (McDonough y Braungart, 2002), el cual es el cimiento esencial del concepto de economía circular.

Hacia 1976, surgió el concepto de economía circuito o lazo (de las palabras en inglés looped economy) (Stahel y Reday, 1976, citado en Geissdoerfer et al., 2017), la cual implica estrategias industriales para la prevención del desperdicio, la creación de trabajo regional, la eficiencia de los recursos, y la desmaterialización de la economía industrial. Detrás del concepto de economía circuito se encuentra la idea de la 'simbiosis industrial' (Stahel y Reday, 1976, citado en Geissdoerfer et al., 2017), la cual representa forma en que los materiales se pueden mover desde la producción hasta el consumo, y luego el desecho; y entonces se convierten en recursos para nuevos procesos, apoyando a la producción nuevamente.

La contracultura ambientalista impulsa por primera vez un movimiento disidente al sistema económico convencional siendo una iniciativa políticamente más comprometida con sus ideales ambientalistas. Aún más importante es el aporte que forma nuevas ideas y soluciones innovadoras ante el dogma económico vigente. La contracultura ambientalista toma tal fuerza en la sociedad, que logra ser escuchada en círculos académicos formales y cúpulas políticas de gran relevancia en el mundo desarrollado y en vías de desarrollo.

En 1972 un equipo de investigadores del Massachussets Institute of Technology publicó el informe conocido como Los límites del crecimiento (Meadows, et al., 1972). Este informe discute la inviabilidad de un crecimiento sin límites en un mundo con escasez. En segundo lugar, se destaca la imposibilidad física del crecimiento indefinido, y se cuestiona abiertamente la deseabilidad del crecimiento económico. Los límites del crecimiento (Meadows, et al., 1972) logra influenciar otros círculos académicos, de la sociedad civil y de la gobernanza política, ambiental y económica global, permeando así en el pensamiento de desarrollo convencional a un nivel nunca visto antes.

Es así como, en un afán de mitigar las consecuencias negativas sobre el ambiente del sistema económico prevaleciente, la Comisión Mundial del Medio Ambiente y el Desarrollo (CMMAD) de las Naciones Unidas, liderada por Gro Harlem Brundlandt, desarrolla el reporte Nuestro futuro común en 1987, enfocándose en la necesidad de administrar y usar los recursos naturales de manera prudente para lograr el principio de la equidad intergeneracional, en donde se "satisfacen las necesidades del presente sin comprometer la capacidad futura para satisfacer sus propias necesidades" (CMMAD, 1987:59).

Pearce y Turner (1990) en su obra Economics of Natural Resources and the Environment aportan por primera vez un acercamiento a la idea de la economía circular. Ellos 
argumentan que la diferencia esencial entre los sistemas económicos y los naturales es que los sistemas naturales tienden a reciclar sus residuos. Ellos usan la primera ley de la termodinámica para indicar que no se puede crear ni destruir energía y materia. Sea lo que sea que se utilice en la forma de recursos, debe terminar en alguna parte del sistema ambiental. Por tanto, ellos insisten en que el planeta Tierra en sí mismo es un sistema cerrado, que se caracteriza por relaciones circulares. Cada elemento es un insumo en algo más. A pesar de esto, no todo residuo es reciclable. Aquí es donde Pearce y Turner se apoyan en la segunda ley de la termodinámica para entender mejor el sistema circular.

La segunda ley de la termodinámica utiliza el concepto de la entropía como una medida de la energía que ya no se puede transformar en trabajo. Según Riechmann (2010), los recursos naturales con baja entropía son los que poseen un alto grado de orden y disponibilidad, tales como los depósitos naturales de carbón y acero, los cuales se han ido agotando rápidamente. Por tanto, la prioridad de la humanidad debería de ser la disminución de la entropía hasta donde sea posible. Según Pearce y Turner (1990), mientras no se desechen cantidades y calidades que no sean conmensurables con la habilidad asimilativa del ambiente, entonces el sistema económico circular no funcionará como un sistema natural asimilando los residuos, y los devolverá al sistema económico; de esta forma entrará en un estado estacionario de forma planificada.

Otro aporte importante que se forma en la contracultura es el de Graedel y Allenby (1995) con la "ecología industrial", que propone una forma en que la humanidad "deliberada y racionalmente" se acerque y mantenga una capacidad de carga deseable en el planeta, dada la evolución económica, cultural y tecnológica (Graedel y Allenby, 1995: 8). Los factores para optimizar son los recursos, la energía y el capital. Estos dos autores enfatizan los procesos industriales y de productos desde una perspectiva dual de competitividad de los productos con interacciones ambientales, llevando a prácticas deseables que pueden lograr un mundo con un estado estacionario sostenible con alta calidad de vida.

Ampliando la explicación del concepto de diseño regenerativo de Lyle (1994), se enfatiza en las prácticas del uso de la tierra industrial y su uniformidad administrada (son patrones simples y formas diseñadas para ser replicadas fácilmente en cualquier lado). Enfatiza que eventualmente un sistema de una vía destruiría los paisajes de los que depende. Por esta razón, el diseño regenerativo es fundamental para reemplazar los sistemas lineales de flujos de producción con flujos cíclicos para recursos, centros de consumo, y repositorios. Este facilitaría el reemplazo continuo, a través de sus propios procesos funcionales, de energía y materiales usados en su operación. Según Lyle (1994), la tecnología regenerativa funciona dentro de los sistemas de flujos que la naturaleza ha desarrollado, usando los medios de la naturaleza para rellenarse sobre una base sostenible, y mantener su integridad funcional, o su capacidad de operar. Los diseños regenerativos siguen los principios básicos del orden ecológico relacionado con la estructura, función y patrón de localización, todos los cuales se desarrollaron y evolucionaron con el tiempo. 
En resumen, autores como Carson (1962), Commoner (1963), Boulding (1966/2012) y Erhlich (1968) nos muestran que el planeta está llegando a su límite de crecimiento y que el estado estacionario podría instalarse abruptamente. Por otro lado, han existido otras iniciativas con un alcance más global, e inclusive con mayor reconocimiento científico, que son los informes de los Limites del crecimiento (1972) y Nuestro futuro común (1987), que han permitido darle mayor credibilidad y mayor alcance político, económico y social al movimiento ambientalista.

Paralelamente otros autores han iniciado la creación de conceptos y esquemas innovadores que construyen un nuevo paradigma del desarrollo como el que representaría la propuesta de la economía circular. Ellos han evolucionado la idea del diseño ecológico tal como lo hace McHarg (1969) con su "diseño natural", y Lyle (1994) con su "diseño regenerativo", así como también Stahel y Reday (1976) con la "economía de circuito" y la "simbiosis industrial". Sustentados en Stahel y Reday (1976), Pearce y Turner (1990) siembran por primera vez la semilla del concepto economía circular, sustentado en las leyes de la termodinámica, logrando explicar la circularidad de los flujos del sistema natural y de sus residuos. Luego Graedel y Allenby (1995) proponen la ecología industrial, en donde explican cómo los procesos industriales pueden simular el comportamiento de la naturaleza, considerando las interacciones con los sistemas circundantes, a saber: la naturaleza. Por tanto, se fortalecen los cimientos del concepto de economía circular para el siglo XXI, en el que alcanzará la madurez de la evolución epistemológica como nuevo paradigma del desarrollo.

\section{Consolidación histórica-epistemológica de la economía circular como nuevo paradigma de desarrollo en el siglo XXI}

Los cimientos conceptuales y epistemológicos creados hasta ahora le dan una gran fortaleza al concepto de economía circular, inclusive como un nuevo paradigma del desarrollo durante el presente siglo XXI, gracias a nuevos aportes de diversos autores.

La renombrada obra de McDonough y Braungart (2002) De la cuna a la cuna (en inglés Cradle to Cradle) consideran que el sistema planetario es un sistema cerrado con recursos valiosos y finitos. Si el sistema económico continúa contaminando y desechando sin esperanza de reutilizar, entonces se podría entrar en un estado estacionario sin calidad de vida. Los humanos deben aprender a imitar al sistema altamente efectivo de flujo de nutrientes y del metabolismo de la naturaleza 'de la cuna a la cuna', en donde el concepto de desperdicio no existe. Esto significa que los nutrientes valiosos contenidos en los materiales le dan forma y determinan el diseño: la forma sigue la evolución, no solo la función.

Para ese mismo año, 2002, Benyus (s.f.) en su obra Biomímica resalta la emulación consciente de las bondades de la naturaleza. En un mundo "biomímico", se manufacturarían las cosas de la forma en que las plantas y los animales lo hacen: usando el sol y compuestos simples para producir fibras, cerámica, plásticos y químicos totalmente biodegradables. El mundo se inspiraría en las formas exquisitas mediante las que los organismos se adaptan a sus entornos y a cada uno de los que allí habitan. 
Tanto McDonough y Braungart (2002) como Benyus (2002) han desarrollado filosofías de diseño que caen en la categoría de biomimícos, al ser inspirados en la naturaleza. De acuerdo con Benyus, estos aportes consisten en un enfoque funcional que utiliza la naturaleza como un modelo para que los humanos emulen; es decir, una perspectiva antropocéntrica. El enfoque biomimíco se deriva necesariamente de una comprensión ecológica de cómo funciona la vida, con un enfoque de sistemas más completo y regenerativo. Con este esquema, se puede lograr un estado estacionario sostenible.

Nuevamente Stahel (2006) amplía su aporte con su obra The Performance Economy. El autor busca diseminar tres objetivos fundamentales con una economía de desempeño: a. creación de riqueza aumentada, b. más trabajos, y c. consumo reducido de recursos (de materiales y energía). Stahel (2006) referencia al trabajo de Paul Romer (1990) en el que cada adelanto tecnológico dará réditos cada vez mayores, lo cual a su vez fomenta mayores niveles de crecimiento económico. La tecnología puede lograr esos avances amparados al desarrollo también de una economía del conocimiento, y se puede convertir en la llave para romper con un estado estacionario insostenible.

Estos avances se ponen al servicio de una economía de desempeño, en la que las estrategias de negocios se enfocan en la administración experta de los activos físicos a través del tiempo, lo que se sitúa dentro de lo que Stahel llama una "economía de circuito o de lazo" (Stahel y Reday, 1976, citado en Geissdoerfer et al., 2017), en la que se acumule la riqueza. En este tipo de economía, el énfasis sería el de administrar eficientemente los activos materiales existentes, y no reemplazar los actuales por unos nuevos.

Pauli (2010) propone la idea de la economía azul, la cual se sustenta en los principios establecidos por Stahel (2006). En esta, la naturaleza regresa a su ruta evolucionaria y a una simbiosis positiva con los sistemas humanos, donde la sociedad se compromete al igual que cada persona por la parte que les corresponde. Pauli argumenta que al apadrinar la física y aplicar el ingenio de la naturaleza, la sociedad hace mejor uso de lo que está disponible a nivel local. Así, todas las necesidades pueden cubrirse, y la sociedad puede operar dentro de la capacidad de carga del territorio en donde se encuentre el proyecto, optimizando la calidad de los comunes que compartimos, fortaleciendo el capital social y promoviendo la resiliencia.

Con estos aportes en la primera década del siglo XXI, se abren las expectativas para que la evolución conceptual y epistemológica de economía circular alcance la robustez y consolidación necesaria que la lleven a ser considerada un nuevo modelo alternativo de desarrollo. La Fundación Ellen Macarthur (FEM) se ha convertido en líder de la sociedad civil global que ha impulsado el desarrollo del concepto de economía circular como uno holístico. En su reporte Towards a Circular Economy: Business Rationale for an Accelerated transition Transition (FEM, 02 de diciembre, 2015) dan una de las mejores definiciones encontradas en la literatura revisada: es restaurativa y regenerativa por diseño, y busca mantener los productos, componentes y materiales en su utilidad y valor máximos todo el tiempo, distinguiendo entre los ciclos técnicos y los biológicos. 
La FEM ha penetrado diversos campos de investigación que se intersecan con otros temas de gran relevancia en la actualidad, en aras de propuestas futuristas para encontrar soluciones. Uno de los que más se resalta es la forma en que los negocios y gobiernos pueden acelerar la transición hacia una economía circular (FEM, 02 de diciembre, 2015), proponiendo el esquema ReSOLVE (de este acrónimo en inglés se detalla el significado de cada letra: Regenerar, dar forma, optimizar, circuito, virtualizar e intercambiar), el cual pretende crear un ciclo revertido. En otros reportes más recientes, la FEM ha encontrado iniciativas amigables con la economía circular en temas como las ciudades y la economía circular (FEM, 24 de enero, 2019 y 04 de marzo, 2019), la economía circular de los alimentos (FEM, 24 de enero, 2019), al igual que otros como la inteligencia artificial y la economía circular (FEM, 23 de enero, 2019), enfocado en las industrias de la alimentación y los productos electrónicos de consumo.

\section{Conclusiones}

Esta revisión literaria permite vislumbrar el pensamiento económico que se ha desarrollado alrededor de la idea de los límites del crecimiento y del estado estacionario, y su relación con los recursos del planeta desde la época por parte de varios de los pensadores clásicos. A su vez, el desarrollo industrial y tecnológico del siglo XX vuelve a situar a la problemática ambiental en un lugar de mucha relevancia para la sociedad, incluso desarrollando un movimiento ambientalista contracultura influyente. La preocupación es tal que autores como Stahel logran proponer el revolucionario concepto de circularidad de la economía. Hacia finales del siglo XX e inicios del siglo $X X I$, diversos autores y organizaciones han dado sentido a perspectivas que han logrado compenetrarse y coexistir unas con otras para formar inclusive un nuevo paradigma alternativo del desarrollo conocido como "la economía circular".

Pensando prospectivamente, se pueden realizar las siguientes recomendaciones sobre cómo implementar la economía circular en nuestras sociedades: a. coordinación de los organismos supranacionales relevantes para motivar a los países en vías de desarrollo a aplicar una economía circular en sus diversos sectores económicos; b. desarrollo de políticas públicas para incentivar prácticas industriales inspiradas en una economía circular; c. implementación de estrategias de desarrollo local en donde los emprendimientos incorporen la economía con empleo decente, con una distribución equitativa de la riqueza creada, y con una fuerte cohesión entre los diversos actores involucrados. 


\section{Referencias}

Benyus, J. (s.f.). Biomimicry: innovation inspired by nature. Nueva York: HarperCollins e-books. https://www.amazon.com/

Boulding, K. (2012). La economía de la futura nave espacial Tierra. Revista de Economía Crítica, 14:327-338. (Obra original publicada en 1966)

Carson, N. A., \& Neill, D. W. (1962). Metabolic abnormalities detected in a survey of mentally backward individuals in Northern Ireland. Archives of disease in childhood, 37(195), 505-513. https://doi.org/10.1136/adc.37.195.505

Carson, R. (2010). Primavera silenciosa. Crítica. (Obra original publicada en 1962)

Commoner, B. (1963). Ciencia y supervivencia. Viking Press.

Comisión Mundial de Medio Ambiente y Desarrollo (CMMAD). (1987). Informe de la Comisión Mundial de Medio Ambiente y Desarrollo: Nuestro futuro común, Documento de la Asamblea General de Naciones Unidas A/42/47. http://www.ecominga.uqam.ca/PDF/BIBLIOGRAPHIE/GUIDE_LECTURE_1/CMMAD-Informe-Comision-Brundtland-sobre-Medio-Ambiente-Desarrollo.pdf.

Crocker, R., Saint, C., Chen, G. \& Yindong, T. (Eds). (2018). Unmaking waste in production and consumption: towards the circular economy (pp. 13-33). Emerald Publishing Limited.

Grupo Banco Mundial. (2020). Estimados de población y proyecciones, indicadores del desarrollo mundial [Fichero de datos]. https://databank.bancomundial.org/reports. aspx?source=world-development-indicators.

Ellen Macarthur Foundation. (2013). Towards the circular economy: Economic and business rationale for an accelerated transition, v. 1. http://www.ellenmacarthurfoundation.org/ business/reports

Ellen Macarthur Foundation. (02 de diciembre, 2015). Towards a Circular Economy: Business Rationale for an accelerated transition. http://www.ellenmacarthurfoundation.org/business/ reports

Ellen Macarthur Foundation. (24 de enero, 2019). Cities and Circular Economy for Food. http:// www.ellenmacarthurfoundation.org/business/reports

Ellen Macarthur Foundation. (23 de enero, 2019). Artificial Intelligence and the Circular Economy. http://www.ellenmacarthurfoundation.org/business/reports

Ellen Macarthur Foundation. (04 de marzo, 2019). Circular Economy in Cities. http://www.ellenmacarthurfoundation.org/business/reports

Ehrlich, P. (1968). The population bomb. Ballantine Books.

Formia, E. (2017). Mediating an ecological awareness in Italy: Shared visions of sustainability between the environmental movement and radical design cultures (1970-1976). Journal of Design History, 30(2), 192-211.

Geissdoerfer, M., Savaget, P., Bocken, N. y Hultink, E. (2017). The Circular Economy: A new sustainability paradigm? Journal of Cleaner Production, 143, 757-768.

Graedel, T., \& Allenby, B. (1995). Industrial Ecology. Prentice Hall.

Hotelling, H. (1931). The economics of exhaustible resources. Journal of Political Economy, 39(2), 137-175.

Jevons, J. S. (2018). The coal question: an inquiry concerning the progress of the nation, and the probable exhaustion of our coal mines. Franklin Classics. (Obra original publicada en 1865). https://www.amazon.com/

Lyle, J. T. (1994). Regenerative design for sustainable development. Wiley. https://www.amazon.com/ 
Malthus, T. (1846). Ensayo sobre el principio de la población (Trad. J. M. Noguera y Joaquín Miguel). Establecimiento Literario y Litográfico de D. Lucas González y Co. (Obra original publicada en 1798). https://archive.org/details/A061288040

Marx, K. (1999). Capital: a critique of Political Economy, Vol. III. International Publishers. (Obra original publicada en 1894). https://www.marxists.org/archive/marx/works/download/pdf/ Capital-Volume-III.pdf

Meadows, D., Meadows, D., Randers, J. y Behrens, W. (1972). Los límites del crecimiento. Informe al Club de Roma sobre el Predicamento de la Humanidad. Fondo de Cultura Económica.

McDonough, W. \& Braungart, M. (2002). Cradle to cradle: Remaking the way we make things. North Point Press. https://www.amazon.com/

McHarg, I. L. (1969). Design with nature. Natural History Press.

Mill, J. S. (1978). Principios de economía política. Fondo de Cultura Económica. (Obra original publicada en 1848).

Organization of Economic Development and Cooperation (2018). Global Material Resources Outlook to 2060: Economic drivers and environmental consequences. OECD Publishing. http://www.oecd.org/environment/global-material-resources-outlook-to-20609789264307452-en.html

Pauli, G. (2010). The Blue Economy. Paradigm Publications.

Pearce, D. \& Turner, R. (1990). Economics of Natural Resources and the Environment. John Hopkins University Press.

Pigou, A. C. (2010). The Economics of Welfare. Liberty Fund, Inc. (Obra original publicada en 1932, cuarta edición). https://edisciplinas.usp.br/pluginfile.php/4154221/mod_resource/content/0/Pigou-The_Economic_of_Welfare_1920.pdf

Polimeni, J. et al. (2009). The Jevons Paradox and the Myth of Resource Efficiency Improvements. Sustainability: Science, Practice and Policy, 15(1):48-54.

Ricardo, D. (2004). On the Principles of Political Economy and Taxation (Edited by Pierro Sraffa). Editorial Liberty Fund. (Obra original publicada en 1821). https://www.econlib.org/library/ Ricardo/ricP.html?chapter_num=6\#book-reader

Riechmann, T. (2010). Entropía, recursos naturales y economía ecológica. Revista Daphnia, 53. http://www.daphnia.es/revista/53/articulo/1028/ Entropia-recursos-naturales-y-economia-ecologica

Roszak, T. (1969). The Making of the Counter Culture: Reflections on the technocratic society and its youthful opposition. California: University of California Press.

Romer, P. (1990). Endogenous Technological Change. Journal of Political Economy, 98(5):71-102.

Smith, A. (s.f.). La riqueza de las naciones. Panamas classics. (Obra original en 1776). https://www. amazon.com/

Stahel, W. (1982). The product life factor. En: Orr, G.S. (Ed.), An Inquiry into the Nature of Sustainable Societies. The Role of the Private Sector (pp. 72-105). Texas: Houston Area Research Center. https://p2infohouse.org/ref/33/32217.pdf

Stahel, W. \& Reday, G. (1976). The Potential for Substituting Manpower for Energy. Report to the Commission of the European Communities.

Stahel, W. (2006). The Performance Economy. Palgrave Macmillan.

Turner, F. (2006). From counterculture to cyberculture: Stewart Brand, the whole earth network and the rise of digital utopianism. Chicago University Press. 